\title{
Well-differentiated fetal adenocarcinoma of the lung: positron emission tomography features and diagnostic difficulties in frozen section analysis-a case report
}

Shuhei Hakiri ${ }^{*}$, Takayuki Fukui ${ }^{1}$, Hideki Tsubouchi' ${ }^{1}$, Ayako Sakakibara², Shingo Iwano ${ }^{3}$ and Toyofumi F. Chen-Yoshikawa'

\begin{abstract}
Background: Well-differentiated fetal adenocarcinoma (WDFA) of the lung is a rare disease that resembles fetal lung tubules. Most of previous reports concerning WDFA have focused on histological features, while there are few reports describing radiological features. In addition, there are no reports evaluating the difficulty of intraoperative diagnosis of WDFA with frozen section. We report a case of WDFA and review the radiological features of WDFA including the findings of F-18 fluorodeoxyglucose positron emission tomography (FDG-PET) and assess the difficulty of intraoperative diagnosis with frozen section.
\end{abstract}

Case presentation: A chest radiography performed in a 20-year-old female revealed a mass in the hilum of the right lung. Computed tomography revealed a well-defined mass measuring $3.5 \times 3.0 \mathrm{~cm}$ in diameter in the right upper lobe, whereas PET showed a high accumulation of FDG. The most likely diagnosis was clinical T2aNOMO stage 1B non-small cell lung cancer. A right $\mathrm{S}^{3}$ segmentectomy was performed via thoracotomy, and a benign tumor that was possibly an adenoma was intraoperatively diagnosed based on frozen section analysis. The mass was a solid tumor measuring $2.9 \times 2.5 \mathrm{~cm}$ in diameter. Microscopically, the tumor comprised abundant glands with single or double layers of nonciliated cells and bronchial structures resembling a fetal lung. Rounded morules of polygonal cells were frequently observed. Immunohistochemistry revealed that nuclei and cytoplasm of the tumor cell were positive for $\beta$-catenin. Finally, the postoperative pathological diagnosis was well-differentiated fetal adenocarcinoma of the lung, and completion right upper lobectomy and mediastinal lymph node dissection were conducted 1 month after the initial segmentectomy. No residual tumor or lymph node metastasis was identified, and the final pathological stage was pT1cNOMO stage $1 \mathrm{~A}$ 3. The patient did not wish to receive any adjuvant therapy. At the 1-year follow-up, no evidence of recurrence was noted.

Conclusions: Here, we report a rare case of well-differentiated fetal adenocarcinoma of the lung that was difficult to diagnose based on radiological evaluations including FDG-PET and intraoperative diagnosis using frozen section analysis. Keywords: Well-differentiated fetal adenocarcinoma, Fluorodeoxyglucose positron emission tomography, Frozen section

\footnotetext{
* Correspondence: h-shuhei-1024@med.nagoya-u.ac.jp

1 Department of Thoracic Surgery, Nagoya University Graduate School of

Medicine, 65 Tsurumai-cho, Showa-ku, Nagoya 466-8550, Japan

Full list of author information is available at the end of the article
}

\section{Springer Open}

( ) The Author(s). 2020 Open Access This article is licensed under a Creative Commons Attribution 4.0 International License, which permits use, sharing, adaptation, distribution and reproduction in any medium or format, as long as you give appropriate credit to the original author(s) and the source, provide a link to the Creative Commons licence, and indicate if changes were made. The images or other third party material in this article are included in the article's Creative Commons licence, unless indicated otherwise in a credit line to the material. If material is not included in the article's Creative Commons licence and your intended use is not permitted by statutory regulation or exceeds the permitted use, you will need to obtain permission directly from the copyright holder. To view a copy of this licence, visit http://creativecommons.org/licenses/by/4.0/. 


\section{Background}

Fetal adenocarcinoma was first described by Kradin et al. in 1982 as a subtype of pulmonary blastoma [1]; thereafter, Kodama et al. introduced the term "fetal adenocarcinoma" in 1984. [2]. In 1998, Nakatani et al. established two case types of fetal adenocarcinoma of the lung: low-grade and highgrade. The low-grade type is known as well-differentiated fetal adenocarcinoma (WDFA) of the lung, predominantly occurring in young females with a good prognosis [3]. Conversely, the high-grade type is poorly differentiated, predominantly occurring among elderly males with a poorer prognosis than the low-grade type. WDFA is a rare disease, accounting for only $0.1 \%$ of all pulmonary neoplasms. It consists of tubules of glycogen-rich, nonciliated cells that resemble fetal lung tubules, and most previous reports concerning WDFA have focused on histological features. Conversely, reports describing the radiological features of this disease are limited $[4,5]$. To the best of our knowledge, only one case has presented the findings of F-18 fluorodeoxyglucose positron emission tomography (FDG-PET) [6]. In addition, no reports evaluating the difficulty of establishing an intraoperative diagnosis of WDFA using frozen section analysis are available. Here, we report a case of WDFA and review the radiological features of this disease, including FDG-PET findings, and assess the difficulty of establishing an intraoperative diagnosis using frozen section analysis.

\section{Case presentation}

A 20-year-old woman with no smoking history presented a round-shaped shadow in the right middle lung field on a chest X-ray during her medical checkup on employment (Fig. 1a), while her chest X-ray 2 years ago had no abnormalities. She exhibited no symptoms and had no comorbidities. Contrast-enhanced computed tomography (CT) of the chest showed a lobulated solid mass in the hilum of the right lung measuring $3.5 \times 3.0 \mathrm{~cm}$ in diameter, which was homogenously enhanced in the early phase scan without any intratumoral fat or calcification identified (Fig. 1b). FDG-PET revealed a high accumulation of FDG in the mass wherein the maximum standard uptake values were 7.1 and 8.4 in the early and delayed phases, respectively (Fig. 1c). The levels of all serum tumor markers of lung cancer such as carcinoembryonic antigen, cytokeratin 19 fragment, and pro-gastrin-releasing peptide were within normal ranges. Based on the radiological findings, the mass was suspected to be either an invasive lung cancer, a lymphoma, Castleman's disease, a solitary fibrous tumor, or an inflammatory pseudotumor. A transbronchial lung biopsy was performed, but the specimen was too small for histological diagnosis. It was decided that the most likely diagnosis of the mass was clinical T2aNOM0 stage $1 \mathrm{~B}$ non-small cell lung cancer as per Union for International Cancer Control (UICC) 8th edition criteria.

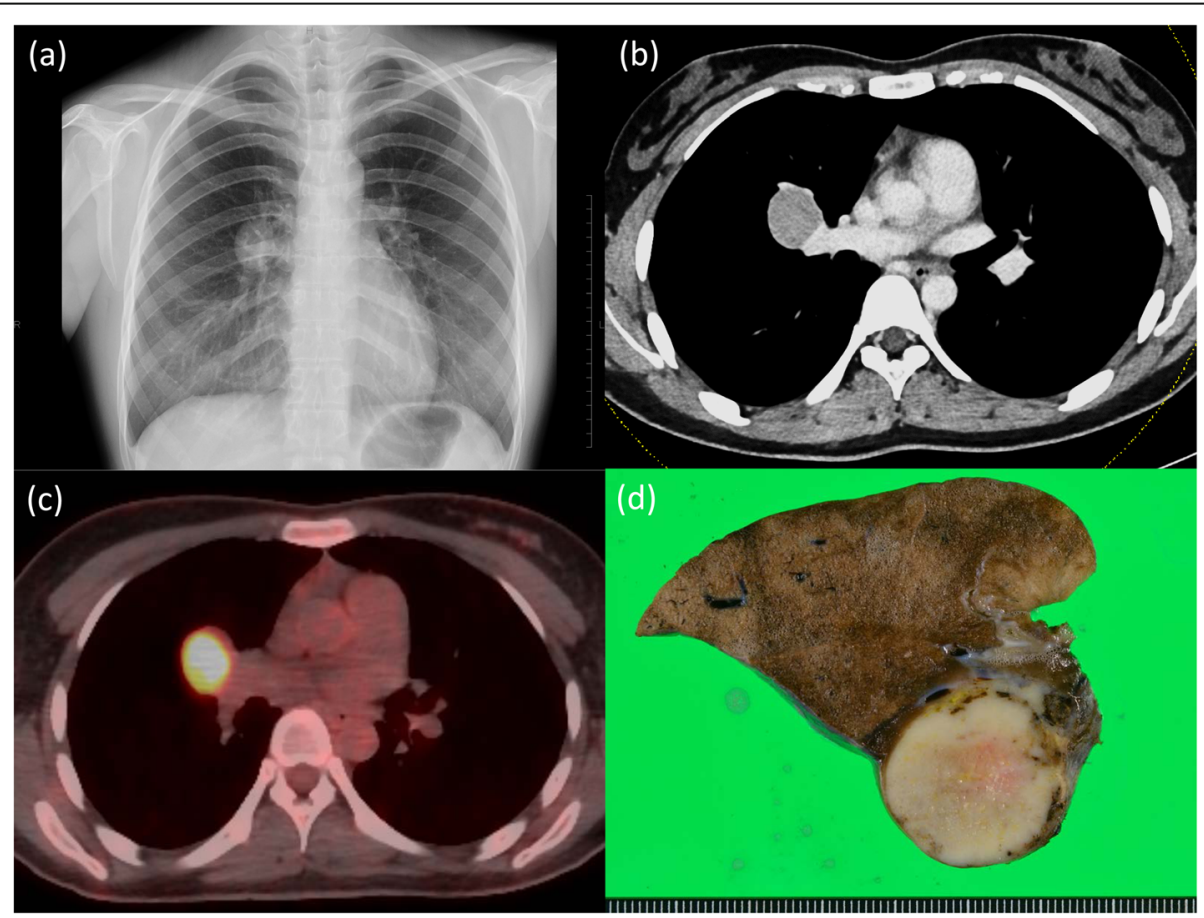

Fig. 1 Radiological findings and macroscopic appearance of the tumor. a Chest X-ray revealed a shadow in the right middle lung field. $\mathbf{b}$ Computed tomography revealed a tumor in the right upper lobe. c F-18 fluorodeoxyglucose positron emission tomography revealed high accumulation of FDG in the lesion of the right upper lobe. $\mathbf{d}$ Macroscopic appearance of the tumor, which appeared as a solitary, white-colored, well-defined, and solid component 
Accordingly, the patient underwent anterolateral thoracotomy through the fifth intercostal space. Intraprocedurally, it was detected that the mass arose from the $\mathrm{S}^{3}$ of the right upper lobe and that its surface facing the interlobular space was covered with visceral pleura. The mass had not invaded the middle lobe, but it was close to the hilar vessels. Thus, wedge resection with the mass was considered to be difficult, and right $S^{3}$ segmentectomy was performed. Intraoperative frozen section analysis revealed that the tumor contained branched gland ducts along with columnar epithelial cells with poor nuclear dysplasia arranged in papillary growth patterns. Therefore, the tumor was diagnosed as a benign pulmonary lesion (e.g., papilloma or adenoma), and the operation was completed without deploying any additional procedures.

Macroscopically, the mass was a solitary, white-colored, well-defined, and solid component measuring $2.9 \times 2.5 \mathrm{~cm}$ in diameter (Fig. 1d). These findings were seldom found in conjunction with necrotic or hematomatous lesions. Microscopically, it consisted of abundant glands with single or double layers of nonciliated cells and bronchial structures that resembled the tubular epithelium of a fetal lung. Rounded morules of polygonal cells with eosinophilic cytoplasm were frequently observed (Fig. 2a). Immunohistochemistry showed that nuclei and cytoplasm of the tumor cells were positive for $\beta$-catenin (Fig. 2b) and negative for p53, nuclei of the tumor cells were positive for thyroid transcription factor 1 , and the cytoplasm was positive for synaptophysin. Based on the abovementioned morphological patterns and immunohistochemical profile, the tumor was finally diagnosed as a WDFA.

At 1 month after the initial operation, the patient underwent completion right upper lobectomy and systematic lymph node dissection. Anterolateral thoracotomy was performed via the fifth intercostal space, same with the incision in the initial operation; further, the lower lobe and chest wall surrounding the wound were widely attached, and both the major and minor fissures were firmly adhered. Although peeling off the adhesions was difficult, the remaining upper lobe was successfully removed without any complications such as vessel injury, lung damage, and massive bleeding. According to the pathological findings, no residual tumor or lymph node metastasis was present, and the final pathological stage was pT1cN0M0 stage 1A3 (UICC, 8th edition). The postoperative course was uneventful, and the patient was discharged on postoperative day 6 . She did not wish to receive any adjuvant therapy. At 1-year after the second operation, the patient was well without any evidence of recurrence.

\section{Discussion}

In the present report, we observed two important clinical issues. First, WDFA showed a high accumulation of FDG on PET. Second, the intraoperative diagnosis of WDFA appeared difficult using histological examination with frozen section analysis.

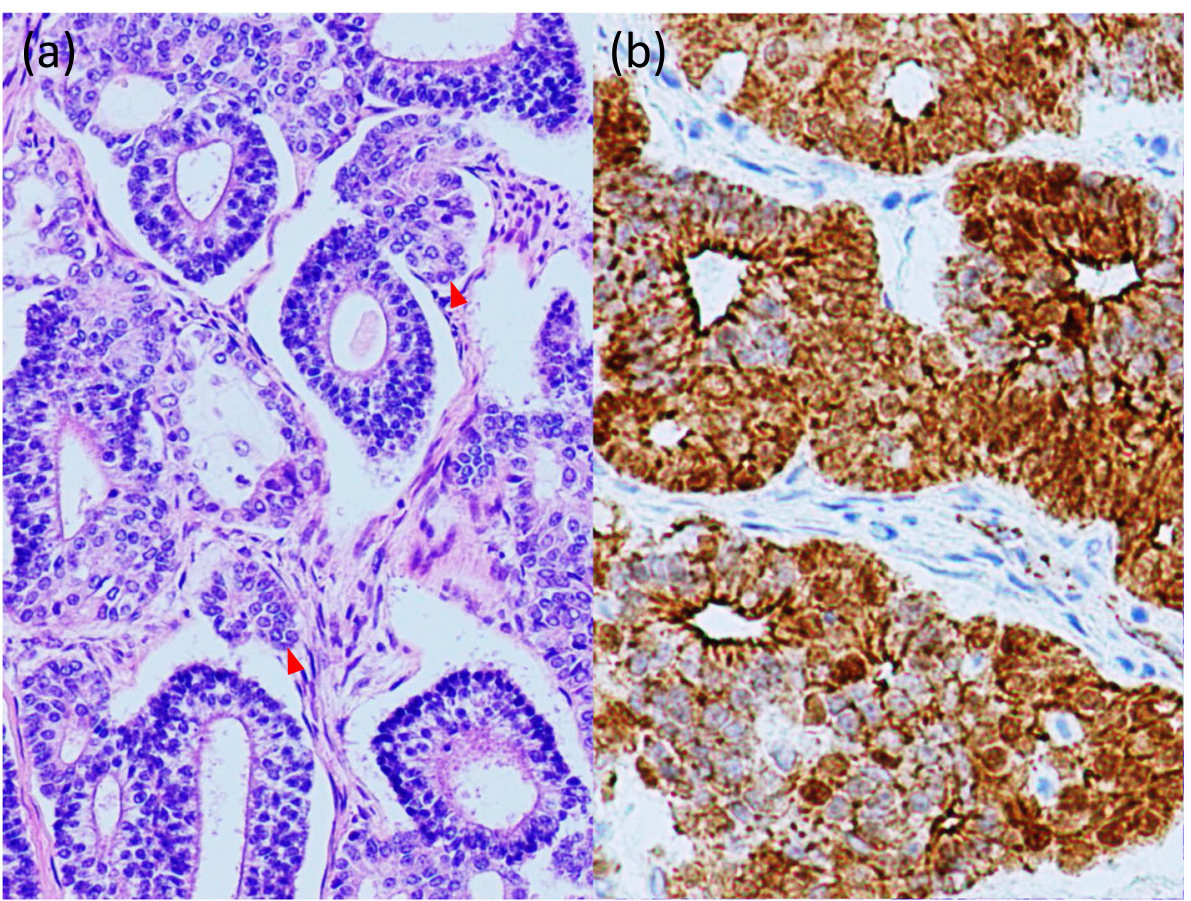

Fig. 2 Microscopic examination of the surgical resection specimen. a Hematoxylin and eosin-stained section showed glands comprising single or double layers of nonciliated cells and some morule formations (red arrowhead). b Immunohistochemical examination showed a positive result for $\beta$-catenin 
Force et al. reported the first case of WDFA with FDG-PET findings [7]. Their case showed a high accumulation of FDG, similar to that observed in our patient. Fetal lung, as opposed to the adult lung, is dependent on glucose metabolism, and fetal lung tissue expresses glucose transporter isoform 1 (GLUT1) [8]. GLUT1 has been reported in most cases of lung carcinoma, particularly those of squamous cell carcinoma; furthermore, in adenocarcinoma, low rates of cell differentiation and larger tumor sizes have been correlated with the expression of GLUT1. WDFA demonstrated a substantial uptake of FDG regardless of the nature of tumor malignancy. It may be difficult to differentiate WDFA from other lung cancers using FDG-PET because of high FDG accumulations on both WDFA and other lung cancers.

In addition, WDFA showed well-defined oval or lobulated solid lesions on contrast-enhanced $\mathrm{CT}$, which are often homogenously enhanced without calcifications. Yamakawa et al. reported that intratumoral enhancing vasculature was noted in the early phase of dynamic CT, whereas a persistent and plateau enhancement was noted in the delayed phase [9]. However, these findings were not specific and similar to those of lung cancer, inflammatory pseudotumor, solitary fibrous tumor, lymphoma, sarcoidosis, and Castleman's disease. Therefore, the preoperative diagnosis of WDFA appears difficult based on radiological findings only.

Furthermore, to the best of our knowledge, no case of WDFA has been diagnosed using intraoperative frozen section analysis, whereas some cases have been diagnosed via aspiration cytology preoperatively. Geisinger et al. reported four cases of WDFA diagnosed using aspiration cytology with immunochemical staining; however, these authors concluded that the cytological diagnosis of WDFA may be challenging [10]. Finally, they proposed that immunochemical studies using markers of epithelial and neuroendocrine differentiation and using $\beta$-catenin will definitely support the diagnosis of WDFA where warranted. Moreover, Proctor et al. suggested that $\beta$-catenin immunohistochemistry, as part of a panel of immunostaining, would aid in this difficult diagnosis in the setting of a limited biopsy, such as fine-needle aspiration biopsy [11]. Notable histological features of WDFA include substantial morule formation morphologically and positive $\beta$ catenin staining during nuclear and cytoplasmic immunohistochemistry; these findings provide important evidences for achieving an accurate diagnosis. It may be difficult to intraoperatively diagnose WDFA via frozen section analysis if only a small specimen is examined and immunostaining is not performed.

Momozane et al. summarized 31 cases of WDFA resected in Japan, and they asserted the difficulty of establishing a definitive diagnosis preoperatively [12]. Among these 31 cases, 10 were histologically diagnosed with malignant tumor preoperatively; nine of these cases were lung cancers, such as adenocarcinoma, squamous cell carcinoma, and small cell carcinoma, whereas the remaining case was diagnosed as pulmonary blastoma including WDFA. A pathologist with little experience may have difficulty confirming WDFA because of the rarity of the condition. However, if patients are young females of approximately 30 years of age showing a high accumulation of FDG on a well-defined solid lung nodule, WDFA should be listed as one of the differential diseases and the necessity of anatomical lung resection and lymph node dissection should be considered. Local recurrence of WDFA following limited resection and lymph node metastasis has been reported [13]. Adjuvant chemotherapy and radiation are unlikely to provide substantial benefit and are thus not recommended [14].

\section{Conclusions}

We found that WDFA is associated with a high accumulation of FDG on PET scan. Moreover, WDFA may be difficult to diagnose using only small biopsy specimens and using frozen section analysis intraoperatively. Thoracic surgeons and pathologists alike should consider WDFA as one of the differential diagnoses when relatively young women demonstrate a well-defined solid lung lesion with a high accumulation of FDG. If there is strong possibility of WDFA, the necessity of anatomical resection should be considered.

\section{Abbreviations \\ WDFA: Well-differentiated fetal adenocarcinoma; FDG- \\ PET: Fluorodeoxyglucose positron emission tomography; CT: Computed tomography; UICC: Union for International Cancer Control; GLUT1: Glucose transporter isoform 1}

\section{Acknowledgements \\ Not applicable.}

\section{Authors' contributions}

SH wrote the manuscript. SH and TF performed the operation. SH, TF, HT, and TC managed the patient's perioperative course. SI assessed radiological findings, and AS diagnosed pathological examinations. TF and TC contributed to the drafting of the manuscript. TC gave the final approval of this manuscript. All the authors read and approved the final manuscript.

\section{Funding}

Not applicable.

Availability of data and materials Not applicable.

Ethics approval and consent to participate Not applicable.

\section{Consent for publication}

Written informed consent for the publication of this case report was obtained from the patient.

Competing interests

The authors declare that they have no competing interests. 


\section{Author details}

'Department of Thoracic Surgery, Nagoya University Graduate School of Medicine, 65 Tsurumai-cho, Showa-ku, Nagoya 466-8550, Japan. ${ }^{2}$ Department of Pathology and Laboratory Medicine, Nagoya University Hospital, Nagoya, Japan. ${ }^{3}$ Department of Radiology, Nagoya University Graduate School of Medicine, Nagoya, Japan.

Received: 12 May 2020 Accepted: 15 June 2020

Published online: 29 June 2020

\section{References}

1. Kradin RL, Young RH, Dickersin GR, Kirkham SE, Mark EJ. Pulmonary blastoma with argyrophil cells and lacking sarcomatous features (pulmonary endodermal tumor resembling fetal lung). Am J Surg Pathol. 1982;6:165-72.

2. Kodama T, ShimosatoY WS, Koide T, Naruke T, Shimase J. Six cases of welldifferentiated adenocarcinoma simulating fetal lung tubules in pseudoglandular stage: comparison with pulmonary blastoma. Am J Surg Pathol. 1984;8:735-44.

3. Nakatani Y, Kitamura H, Inayama Y, Kamijo S, Nagashima Y, Shimoyama K, et al. Pulmonary adenocarcinomas of the fetal lung type: a clinicopathologic study indicating differences in histology, epidemiology, and natural history of low-grade and high-grade forms. Am J Surg Pathol. 1998;22:399-411.

4. Furuya $\mathrm{K}$, Yasumori $\mathrm{K}$, Takeo S, Uesgi N, Otsu Y, Ichiki M, et al. Welldifferentiated fetal adenocarcinoma of the lung: early-phase sequential high-resolution computed tomographic findings. J Comput Assist Tomogr. 2008;32:806-9.

5. Yamakawa T, Nambu A, Kato S, Kawamoto M, Fujino S, Watanabe M, et al. Well differentiated fetal adenocarcinoma of the lung in a 38-year-old woman: dynamic computed tomography findings. Jpn J Radiol. 2013;31: 143-7.

6. Douglas EP, Jazbieh M, Neil K, Alex GL, Samuel AA. Positron emission tomography in well differentiated fetal adenocarcinoma of the lung. Clin Nucl Med. 2006;31:213-4.

7. Force S, Patterson GA. Clinical-pathologic conference in general thoracic surgery: pulmonary blastoma. J Thorac Cardiovasc Surg. 2003;126:1247-50.

8. Ito T, Noguchi Y, Udaka N, Kitamura H, Satoh S. Glucose transporter expression in developing fetal lungs and lung neoplasms. Histol Histopathol. 1999;14:895-904.

9. Geisinger KR, Travis WD, Perkins LA, Zakowski MF. Aspiration cytomorphology of fetal adenocarcinoma of the lung. Am J Clin Pathol. 2010;134:894-902.

10. Proctor L, Folpe AL, Esper A, Wolfenden LL, Force S, Logani S. Welldifferentiated fetal adenocarcinoma of the lung: cytomorphologic features on fine-needle aspiration with emphasis on use of beta-catenin as a useful diagnostic marker. Diagn Cytopathol. 2007:35:39-42.

11. Momozane T, Kusumoto H, Ohse N, Hayashi A, Takeuchi Y, Maeda H. A resected case of well-differentiated fetal adenocarcinoma of the lung in a young male adult. Jpn J Chest Surg. 2012;26:746-52.

12. Atoini $F$, Ouarssani $A$, Elmejereb $C$, Hachimi MA, Jaafari $H$, Aitlhou F, et al. A well-differentiated fetal adenocarcinoma of the lung with early local recurrence after limited resection. Thorac Cancer. 2011;2:123-7.

13. Politiek MJ, Vrugt B, Aalbers R. A 49-year-old woman with well-differentiated fetal adenocarcinoma. Neth J Med. 2001;58:177-80.

14. Osakwe NC, Tong J, Rodgers WH, Kansler AL, Lee PC, Paul S. A rare case of fetal adenocarcinoma of the lung. Ann Thorac Surg. 2014;98:2217-8.

\section{Publisher's Note}

Springer Nature remains neutral with regard to jurisdictional claims in published maps and institutional affiliations.

\section{Submit your manuscript to a SpringerOpen ${ }^{\circ}$ journal and benefit from:}

- Convenient online submission

- Rigorous peer review

- Open access: articles freely available online

- High visibility within the field

- Retaining the copyright to your article

Submit your next manuscript at $\boldsymbol{\nabla}$ springeropen.com 